\title{
DEVELOPMENT OF A TACTILE AND SLIP SENSOR CONTROLLED PROSTHETIC HAND SYSTEM
}

\author{
W. Xu' ${ }^{1}$, R. C. Wang ${ }^{2}$, J.C. Zhang ${ }^{2}$ and D. W. Jin ${ }^{2}$ \\ ${ }^{1}$ School of Engineering and Physical Sciences, Stag Hill, Guildford, UK \\ ${ }^{2}$ Department of Precision Instruments and Mechanology, Tsinghua University, Beijing 100084, China
}

\begin{abstract}
Supported by the latest sensor and microcontroller technologies, prosthetic hands have been widely used to reclaim the human functionaries. Among these, the most advanced prosthetic hand was controlled by the tactile and EMG singles. However, for a slippery object, attention has to be taken for the inexperienced users who need to control the shrinkage of the wrist flexor carefully. In this paper, the authors presented a prosthetic hand control system using PVDF film sensor to provide both tactile and slip force feedback signals to operate the hand. The PVDF film sensor used for this control system was specifically developed to detect both tactile and slip force between the prosthetic finger and object. The method of distinguishing two signals was described. A prototype system was constructed using a microcontroller to process the signal from the sensor and provide control signal to the motors operate the prosthetic hand. The test result of the prototype device shown that comparing with the one without slippery signal feedback, this system was easy to operate for an inexperienced user.
\end{abstract}

Key words: Prosthetic hand, slip sensor, microcontroller and stepper motor

\section{Introduction}

Since 1980s, prosthetic hands have been widely used to regain functionalities for the hand amputation patients [1\&2]. The latest technologies were used by prosthetic hand to achieve human hand's closing and opening and the rank of the gripping strength using tactile sensor and electromyography (EMG) signal, and microcontroller controlled stepper motor system. The Ottobock SUVA automatic touch hand represented the latest technology of using tactile sensor and EMG signal to control a prosthetic hand [3].

Though the SUVA automatic touch hand can detect the tactile force and grip a delicate and frangible object, it is unable to detect the slipping force between the prosthetic fingers and object. In order to grip a slippery and frangible object, obviously the ability of slippery sensing and control signal feedback is essential to the prosthetic hands.

Considerable effort has been made to achieve both tactile and slippage detections [4\&5]. For instance, Dario, et al [5] used PVDF developed a sensor with a matrix of 128 sensing elements for robot and feedback and gripping force. This method involved enormous calculations to process the signal from the sensor. This may not be a critical issue for the industrial robot which is normally supported by a workstation and complicated hardware. However, the calculations involved are beyond the process ability of a microcontroller used by a prosthetic hand. To overcome this problem, the authors developed a tactile-slip sensor with simple detective mechanism and structure to control the prosthetic hand.

The tactile-slip sensor developed by the authors used PVDF film as sensing element. The fundamental principle of detecting touch and slippage between object and prosthetic finger was based on analyzing and differentiating the signal pattern generated under two different conditions. The PVDF film was used not only because it had high effect and piezoelectricity voltage constant, but also it could be fitted to a curved surface of the prosthetic finger. For slippage detection, a rubber membrane with designated pattern was used to generate mechanical vibration hence alternating signal by the PVDF film. This sensor had the advantageous of simple structure, high response speed and short retardation time. The experiments were carried out on a prototype system. The result shown that the signal variance directly corresponding to the material and surface roughness of the object and the slippage generated alternating signal was differentiated from the tactile signal.

\section{Method and Materials}

\section{Design of the PVDF tactile and slip sensor}

The tactile-slip sensor consists of $50 \mu \mathrm{m}$ PVDF film and a layer of rubber membrane. In order to improve signal sensitivity, double layered film was used to construct the sensor. On the outer surface of the rubber membrane there was designated strip or spot patterns, which were used to trigger small mechanical vibration when a slippage took place. The pattern can be arranged in a different format such as straight strip, circular strip and spot array. In this study, the spot pattern was used and arranged perpendicular to the axis of the prosthetic figure. The distance between each spots was $0.8 \mathrm{~mm}$.

Two signal wires were connected to the positive and negative poles of the PVDF film respectively by means of conductive resin DTA- 40 . The electrodes that made by copper plate and enamel wires were used as transitional leading wire 
from the sensor to the low noise signal cable. The sensor was curved to cover a prosthetic fingertip made by $\Phi 13 \times 15 \mathrm{~mm}$ aluminum tubing. The PVDF film and rubber membrane were fixed to the finger at two ends using $12 \times 3 \times 1 \mathrm{~mm}$ aluminum strips. Fig. 1 shows the structure of the tactile-slip sensor.

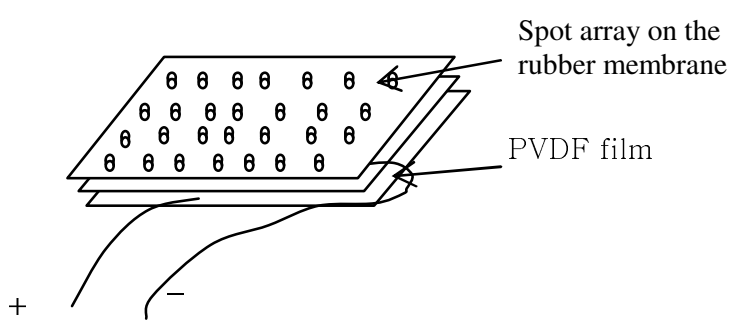

Fig.1 A PVDF tactile and slip sensor

As shown in the Fig. 2, the finger column was mounted on the frame of prosthetic hand and a thin silicon rubber layer was used to cover the sensor part of prosthetic thumb. The enamel wires were connected to a $2 \mathrm{~mm}$ diameter low noise cable at the mounting point. The other end of the cable was connected to a conventional data acquisition system and signal process unit.

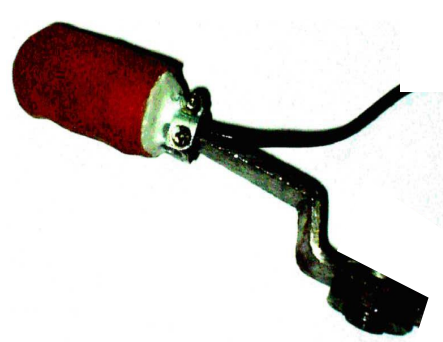

Fig.2 Prosthetic thumb with sensor

\section{Principle of the force detections}

The block symbol in Fig. 3 shows an energy transformation model of a PVDF film. With two plated electrodes attached to its upper and lower surfaces, a capacity sensor is formed. It can produce charge in the case of the stress acting on the micro unit varieties. The equation 1 defines the charge produced in the unit area of PVDF film.

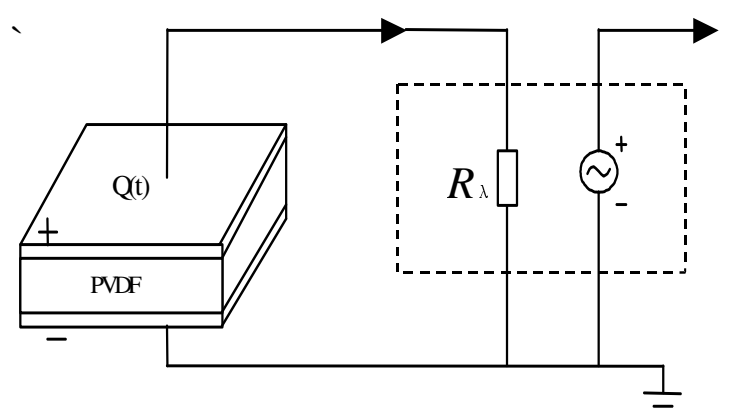

Fig.3 Energy model of the PVDF sensor

$$
\Delta q=\sum_{j=1}^{3} d_{3 j} \Delta \sigma_{j}
$$

where $\triangle \sigma_{\mathrm{j}}$ represents the variational amount of the stress in each direction; $d_{3 j}$ are the piezoelectricity constants in each direction.

To connect an amplifier with input resistance $\mathrm{R}_{\lambda}$, the dynamic equation of piezoelectric effect can be represented by the equation 2 .

$$
Q(t)=\sum_{j=1}^{3} d_{3 j} \Delta \sigma_{j}(t)-\int_{t_{0}}^{t} i d t
$$

where $i \approx \frac{Q(t)}{R_{\lambda} \cdot C}$ is the leaking current. If the initial area density in one of the points of the sensor is $q\left(x, y, t_{0}\right)$, the charge density at time $t$ is given by equation 3 .

$$
q(x, y, t)=q\left(x, y, t_{0}\right)+\int_{0}^{t}\left[\sum_{j=1}^{3} d_{3 j} \frac{\partial \sigma(x, y, t)}{\partial t}\right] d t
$$

Then the charge in area $\Omega$ of the PVDF film is given by equation 4 .

$$
Q(t)=Q\left(t_{0}\right)+\iint_{\Omega}\left[\int_{0}^{t}\left[\sum_{j=1}^{3} d_{3 j} \frac{\partial \sigma(x, y, t)}{\partial t}\right] d t\right] d x d y
$$

After attach the sensor to the amplification system, leaking current should be taken into account. Then the Eq. 4 can be written as equation 5.

$$
\begin{aligned}
& Q(t)=Q\left(t_{0}\right)+\iint_{\Omega}\left[\int_{0}^{t}\left[\sum_{j=1}^{3} d_{3 j} \frac{\partial \sigma(x, y, t)}{\partial t}\right] d t\right] d x d y \\
& -\int_{t_{0}}^{t} \frac{Q(t)}{R_{\lambda} \cdot C} d t
\end{aligned}
$$

Under compression force, the main contribution of piezoelectric effect of the PVDF is in the direction perpendicular to the film surface. For simplification, if only takes the vertical force $\left(\mathrm{d}_{33}\right)$ acting on PVDF into account and assuming the stress change in the sensory area is uniform. Eq. 5 can be written as:

$$
Q(t)=\Omega \cdot d_{33} \cdot \exp \left(-t /\left(R_{\lambda} \cdot C\right)\right) \cdot U(t)
$$

The Eq. 6 indicates that the quantity of charge will decreased progressively following the time constant $R_{\lambda} \cdot C$ according to exponential law. 
This is caused by current leaking of the amplifier and the sensor. In addition, from the Eq.1 that the quantity of charge is proportional to the strain produced from the PVDF; thus the relation between the tactile force and the peak value is nearly linear. In view of this the value of the tactile force can be written as:

$$
|F|=K|U|
$$

From the detected characteristic of $\mathrm{U}$, the tactile signal and slipping signal can be identified. For a tactile signal, from Eq.6, it can be found that the peak value of the signal is attenuated according to exponential law. In the case of a slippage occurs between the object and finger, the mechanical vibration could be generated, which alters the stress field in the PVDF film. As the result of this, an alternating signal could be induced to represent mechanical form of a series of contact and releases. This distinguished feature was used to identify a slippage between the prosthetic finger and object.

\section{Construction of the control system}

Fig. 4 shows the main circuit diagram of the control system. It consisted of an Intel's 8751 microcontroller and an ADC0804 A/D chip. The $\mathrm{ADC0804}$ is a CMOS 8-bit successive approximation $\mathrm{A} / \mathrm{D}$ converter using a resistive ladder and capacitive array together with an auto-zero comparator. In this prosthetic and control system one A/D channel was used as the tactile and slip signal were distinguished using the same signal from the sensor. ADC0804's 8 bit A/D accuracy was enough because the A/D converter only needed to provide an overall signal pattern from the PVDF sensor to the microcontroller. The timer of the ADC0804 was connected to the ALE of the microcontroller at a frequency of $600 \mathrm{kHz}$. This was to maintain the microcontroller operated at a 6 times higher frequency which was $3600 \mathrm{kHz}$

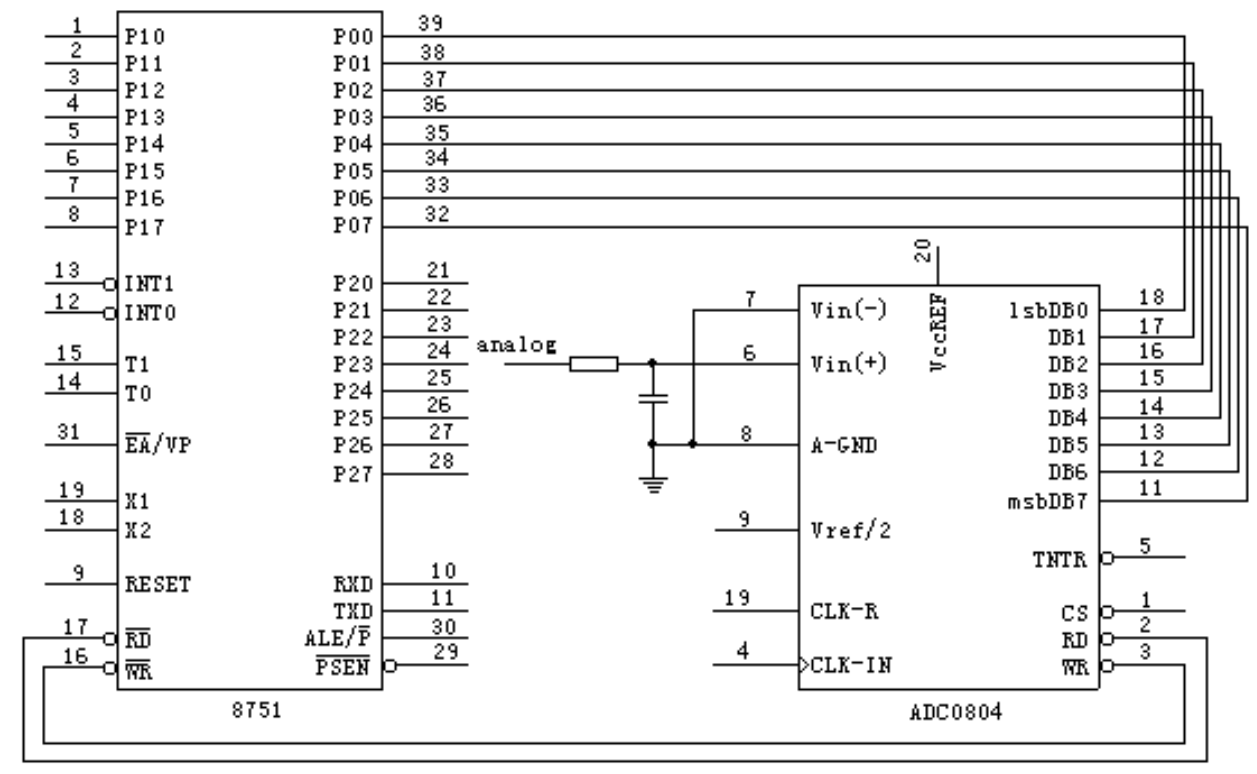

Fig.4 Diagram of the control system

Intel 8751 is a 40 pin microcontroller with one CPU, two timers, 128 bytes of RAM, 4 kBytes of EEPROM, four 8-bit biderectional I/O ports and an EIA-232 port. It was programmed to identify the tactile and slippage contact between prosthetic hand and object. Through its I/O ports, 8751 also produced digital output to the stepper motor to control the operation of the prosthetic hand. Fig. 5 shows the control unit of the prosthetic hand. In order to achieve a reliable control and reduce the disturbing of from other signals, signal conditioning and standard EMG signal filter were used.

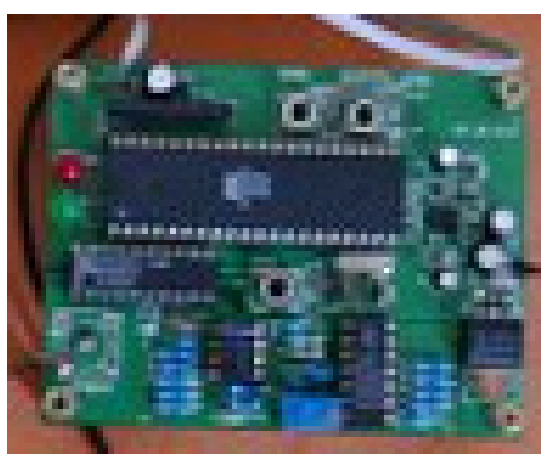

Fig. 5 Control unit of the prosthetic hand 


\section{Experiment and Result}

Experiment setups

To assess the prototype device of the tactile and slip sensor, three experiments were set up. A minimum response threshold value was set to the feedback signals from the sensor. The threshold value of the tactile was set as $7 \mathrm{~N}$ and the variance response threshold value for slippage detection was 0.002. This figure was obtained from the experiment and varied as the object material changes.

Firstly, the tactile function of the sensor was tested by applying various forces to the prosthetic finger. The force range of the test was between 1.8 to $7.5 \mathrm{~N}$. This force range was selected based on the output sensitivity of PVDF film, loading accuracy of the test set up, and the control system used to obtain tactile response curves of the sensor.

The second experiment was set up to test the detection of the slippage. The tests were carried out under constant touching force. At a $0.2 \mathrm{~N}$ touching force, a slippage between prosthetic finger and object was introduced to test the response of the sensor. Different materials were selected for the test and alternating response curves were obtained. For all slippage tests, the motion directions of the slippage were parallel to the rubber membrane.

The last experiment was carried out using an EMG controlled prosthetic forearm. The tactile-slip sensor was mounted on the prosthetic thumb. It consisted of prosthetic hand, tactile-slip sensor, and control system with imported EMG signal. To control the griping operation of the prosthetic hand, EMG signal from muscle, feedback signal from both tactile and slippage detection were used. The EMG signal was used to operate the close and open of the hand, the tactile detection feedback was used to control the griping force make sure the hand was closed adequately neither too tight nor too loose. At the meanwhile, the slippage feedback signal was used to increase the griping force if a slippage was detected.

\section{Results of the tactile response}

Experiment was carried to assess the tactile function of the prosthetic thumb mounted with PVDF film sensor. Fig. 6 shows the voltage response of the sensor to different tactile releasing forces from 2 to $7.5 \mathrm{~N}$. The values were obtained from an average of three measurements. The straight line gives the least squares representation of the relationship between tactile force and amplitude of the voltage response from the PVDF film sensor.

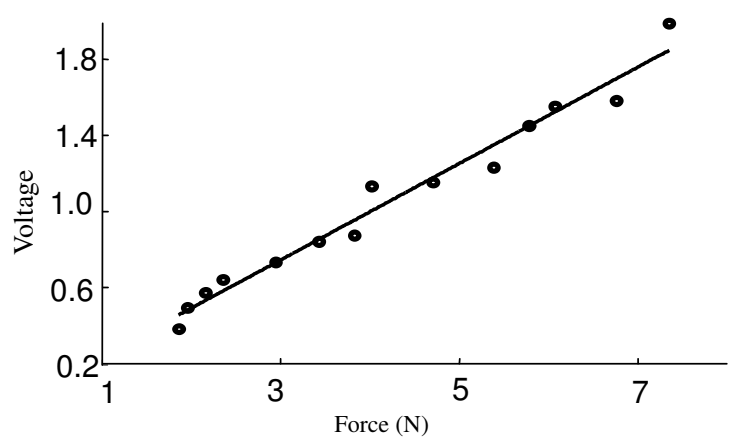

Figure 6 Amplitude of tactile

Table 1 shows the ratio between force and voltage amplitude of the sensor signal. A liner relationship can be clearly observed between load and signal response. The corresponding Eq.7 in the previous section also confirmed the theoretical linear relationship.

Table 1..Relationship between peak voltage and applied pressure load

\begin{tabular}{llllllll}
\hline Force $(\mathrm{N})$ & 2.00 & 2.40 & 3.50 & 4.10 & 4.80 & 6.90 & 7.50 \\
\hline Voltage $(\max )$ & 0.500 & 0.650 & 0.850 & 0.770 & 1.160 & 1.590 & 2.000 \\
Ratio & 25.000 & 27.083 & 24.286 & 27.805 & 24.167 & 23.043 & 26.667 \\
\hline
\end{tabular}

It should notice that restricted by the accuracy of the experiment setting up, a precise tactile force measurement was unable to be carried out when the loads were below $2 \mathrm{~N}$. This, however, should not prevent authors to claim this worthwhile work as the general trend from the result confirms with theoretical prediction made in the previous section.

Result of the slippage detection

Fig. 7 (a) and (b) shows typical responses (in 0.8 second) of the tactile-slip sensor under tactile and slip condition respectively. For tactile touch, the response of the sensor appeared to be a single peak high amplitude signal. However, when slippage occurred, the response of the sensor appeared to be an alternating low amplitude signal. The comparison between Fig. 7 (a) and (b) shows that the responses of the sensor to tactile and slippage were significant different. They could be identified within 0.1 to 0.15 second of the slippage through the detection of alternating signal. 


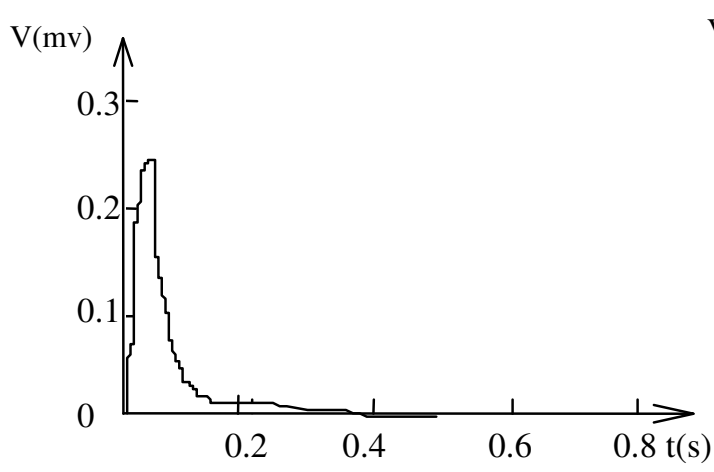

(a) Tactile response curve

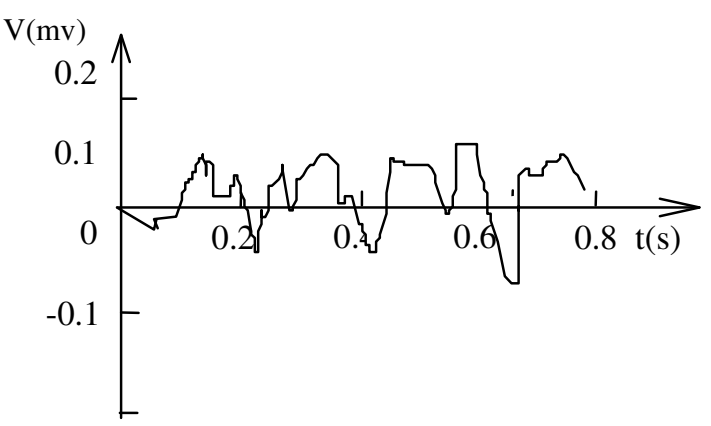

(b) Slippage response curve

Fig.7 Response signals of the tactile and slip sensor

It was obviously that the period of the alternating signal mainly depended on the speed of slippage, the geometrical feature of the surface pattern of the sensor and response of the PVDF film. The response speed of the slippage detection can be increased by refine the surface pattern of the sensor. Therefore the time required to identify the slippage could be reduced further.

Compared to the tactile signal, the slippage response was different in both pattern and amplitudes. The detected signal shown in Fig. 7 (b) was a typical one produced by friction induced micro vibration on the designated surface pattern attached the PVDF film. The alternating feature of the slip signal could be used to detect the occurrence of the slippage and the amplitude of the alternating signal was found related directly to the friction coefficient between the object and the prosthetic thumb, as well as the magnitude of the normal loading to the surface of the object.

Table 2 shows the result of variance of signal when slippage occurred at $0.8 \mathrm{~s}$ using different materials of the object surface. They were obtained under a constant normal force of $0.2 \mathrm{~N}$ to the surface of the object. The variance in the table 2 was calculated using equation 8 .

$$
\sigma_{x}^{2}=\frac{1}{N-1} \sum_{n=0}^{N-1}\left(u_{n}-\bar{u}\right)^{2}
$$

where $u_{n}$ is the output signal, $\bar{u}$ is average of signal, $\sigma_{x}$ is variance of the signal. Sampling time was $0.8 \mathrm{~s}$, sampling rate was $500 \mathrm{~Hz}$.

Table 2. Different material surfaces signal variance when slippage occurred at $0.8 \mathrm{~s}$

\begin{tabular}{lrrrrr}
\hline Material & Plexiglass & $\begin{array}{c}\text { Surface of } \\
\text { xylon table }\end{array}$ & $\begin{array}{c}\text { Engineering } \\
\text { plastics }\end{array}$ & $\begin{array}{c}\text { Common } \\
\text { paper }\end{array}$ & $\begin{array}{c}\text { Smooth steel } \\
\text { pipe }\end{array}$ \\
\hline Variance & 0.0156 & 0.0052 & 0.0041 & 0.0029 & 0.0027 \\
\hline
\end{tabular}

Experiment on EMG controlled prosthetic hand

The experiment using EMG controlled prosthetic hand was divided out in three phases. The first phase was to instruct the prosthetic hand to close its fingers. There was no tactile and slippage feedback signals at this stage until the prosthetic finger touch the object. The EMG signal from the shrinkage of the wrist flexor was used to control the positive rotation of the stepper motor, which closed the prosthetic fingers. When the prosthetic finger and object was in contact and tactile signal was detected by the sensor mounted on the thumb, the control of the close of the prosthetic finger was hand over to tactile force feedback.

The second phase started when tactile signal was detected. As the result shown in the above section, the amplitude of the tactile signal corresponded to the gripping force applied on the prosthetic finger. A tactile threshold was preset to control the gripping force. When the amplitude of tactile signal reached to this value, the positive rotation of the motor stopped, there was no further close of the prosthetic fingers and the object was under this threshold gripping force.

The third phase started when a lifting force was applied to the prosthetic hand and at the meanwhile there was a slippage if the gripping force was insufficient to hold the weight of the object. In this case, the sensor detected the existence of the slippage signal. This signal switched the operation mode of the prosthetic hand back to the phase two and triggered the positive rotation of the motor to grip the object further. This process was repeated until there was no further slippage signal could be detected. The reverse 
operation of the prosthetic hand was controlled by EMG signal of the wrist extensor shrinks. The open of the prosthetic finger was only controlled by the shrinkage of the wrist extensor.

Experiments of the EMG controlled prosthetic hand were carried to grip eggs and wet glass cup using an EMG controlled prosthetic forearm. The experiment showed the slip force detection could be performed in such an "instant" time that the human being would hardly be noted. In the experiments, the hand could lift a wet class cup smoothly and could also hold a fragile object as shown in Fig.8.

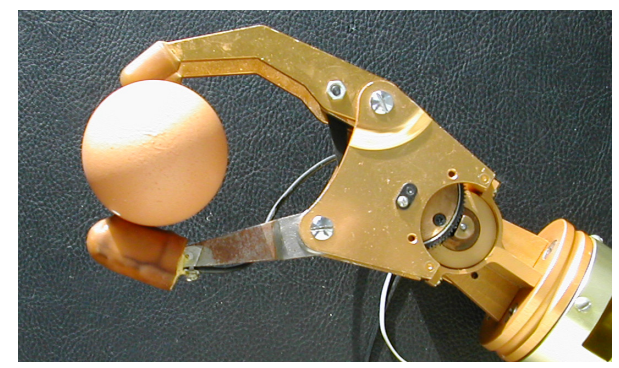

Fig.8 Grab an Egg

\section{Conclusions}

A tactile-slip sensor used by the authors was to provide control feedback to a prosthetic hand. The detection of slippage was achieved using a rubber membrane with designated pattern. It has the advantages of simple structure, convenient signal process and can carry on real-time feedback and control.

An energy transformation model of a PVDF film used for the sensor was derived. In the case of a slippage occurred, the mechanical vibration would be generated. As the result of this, an alternating signal was induced to represent mechanical form of a series of contact and releases. This distinguished feature was used for the sensor to identify a slippage hence control the slippage.

Three experiments were carried out by the authors to evaluate overall performance of the control system. The tactile response test showed that there was a linear relationship between tactile force and amplitude of the tactile signal. This confirmed with energy transformation model derived by the authors. The slippage experiments showed some obvious variances of the response signal. The test proved a slippage could be identified within 0.1 to 0.15 second of the slippage took place through the detection of the alternating signal. The experiments using EMG controlled prosthetic hand were carried out. The objective of the experiment was to assess the usability of the control system. The result showed, with slippage detection, the EMG controlled prosthetic hand was easy to use and able to grab fragile and slip object. Finally, future study has been considered to identify different surface conditions of the objects using touching then adopt control approach..

\section{Acknowledgements}

The authors wish to acknowledge the support of this work by the national Hi-Tech Research and Development (863) Program (№2001AA320601) 、 the Key Program of NSFC (On new generation humanoid prosthesis hand system and its theory) and SRFDP(№20010003060).

\section{Reference}

[1] Jiang, ZW, Chonan, S, Tanahashi, Y, Tanaka, M, Kato, T, Development of soft tactile sensor for prostatic palpation diagnosis: sensor structure design and analysis, Shock and Vibration, 12

[2] Lee, MH, Nicholls, HR, Tactile sensing for mechatronics - a state of the art survey, Mechatronics, 125

[3] SUVA Automatic Touch Hand, Ottobock product catalogue, www.ottobock.com

[4] Melchiorri, C, Slip detection and control using tactile and force sensors, IEEE-ASME Transactions on Mechatronics, 29

[5] Fiorillo, A. S.; Dario, P.; Bergamasco, M. Sensorized Robot Gripper, Robotics, v 4, n 1, Mar, 1988, p 49-55 